\title{
Increasing smoking intensity is associated with increased disease activity in axial spondyloarthritis
}

\author{
Sizheng Zhao ${ }^{1,2} \cdot$ Benjamin Challoner $^{1} \cdot$ Mohammed Khattak $^{2} \cdot$ Robert J. Moots $^{1,2}$ \\ Nicola J. Goodson ${ }^{1,2}$
}

Received: 18 August 2016 / Accepted: 22 October 2016 / Published online: 4 November 2016

(c) The Author(s) 2016. This article is published with open access at Springerlink.com

\begin{abstract}
A history of ever-smoking appears to be associated with a more severe disease phenotype in axial spondyloarthritis (axSpA). However, evidence is sparse for the effect of increased smoking exposure on disease outcomes or whether smoking reduction or cessation improves outcomes. The aim of this study was to explore whether a dose-response relationship exists between pack-years and disease activity and functional impairment in axSpA. Consecutive patients meeting ASAS criteria for axial SpA were recruited from a spondyloarthritis service. The associations between pack-years of smoking and: (1) disease activity (BASDAI/ASDAS), (2) spinal pain, (3) functional impairment (BASFI) and (4) inflammatory markers were explored using multivariable linear models, adjusted for age, gender and use of TNF inhibition (TNFi) therapy. Pack-years were categorised into four groups $(<10,11-20,21-40,>40)$ and analysed with light smoking $(<10)$ as reference. Two hundred and thirty-eight axSpA patients were recruited: $76 \%$ were male, mean age 46.4 years ( $\mathrm{SD} \pm 13.7$ ), and $33 \%$ were treated with TNFi. One hundred and twelve patients reported history of ever-smoking with median pack-year 20 [IQR10-30]. Compared to light smokers, those with higher categories of smoking exposures had higher BASDAI (21-40 pack-years, $\beta=1.6$ (95\% CI 0.28, 2.95); $>40, \beta=2.6(0.54,3.56))$, higher BASFI $(21-40, \beta=2.1$ $(0.42,4.80) ;>40, \beta=3.2(0.76,5.71))$, and higher ASDAS $(21-40, \beta=0.82(0.14,1.51))$. This cross-sectional study
\end{abstract}

Nicola J. Goodson

ngoodson@liverpool.ac.uk

1 Academic Rheumatology Department, Clinical Sciences Centre, Aintree University Hospital, Liverpool, UK

2 Institute of Ageing and Chronic Disease, University of Liverpool, Liverpool, UK demonstrated that smoking is associated with increased axSpA severity markers in a dose-response manner. Particular effort should be made to restrict smoking exposure early before accruing a significant number of pack-years.

Keywords Axial spondyloarthritis · Ankylosing spondylitis · Cigarette smoking · Disease activity · Functional impairment

\section{Introduction}

Axial spondyloarthritis (axSpA) is a chronic inflammatory disease involving the entheses. Musculoskeletal involvement is predominantly axial (sacroiliitis, spondylitis) leading to inflammatory back pain, but can be also involve peripheral joints and tendons (arthritis, enthesitis, dactylitis). AxSpA can be further divided into 'radiographic' (ankylosing spondylitis, AS) and 'non-radiographic' (nraxSpA), depending on whether definitive structural changes are evident on plain radiographs of sacroiliac joints [1, 2]. Patients with nr-axSpA may display active inflammation on magnetic resonance imaging (MRI). Nr-axSpA may represent early AS but may also be a limited form that is symptomatically similar but does not lead to structural changes. Since delineation of nr-axSpA and AS is artificial and unreliable, both forms have been suggested to be one disease [3].

Cigarette smoking appears to have an important role in axSpA. Studies have demonstrated that smoking is associated with incident AS [4] and earlier onset of axSpA [5]. Smokers with AS have increased disease activity, functional impairment and reduced quality of life [6, 7]. The reasons for these associations are not well understood but may reflect increased inflammatory burden associated 
with smoking or possibly confounding effects of socioeconomic class. However, previous studies exploring association between smoking and disease severity have utilised cross-sectional study designs, which limit causal inference. Exploring the dose-response relationship between smoking and disease severity may provide more evidence for causal relationship. Existing studies have mostly been in small cohorts with varying results and many methodological limitations [8-13]. The aims of this study were to test the hypothesis that pack-years of cumulative exposure to cigarette smoking in ever-smokers are associated with disease activity and functional impairment in axSpA and to quantify its effect size.

\section{Method}

Consecutive patients attending a tertiary referral spondyloarthritis service, in a UK hospital setting, were recruited between September 2010 and December 2015. Patients were included if they fulfilled the ASAS criteria for axial SpA [2].

Clinical data were collected during routine out-patient assessments. Patient characteristics were recorded including: age, gender, body mass index (BMI). Smoking status was categorised as current smoker, ex-smoker and nonsmoker. For patients who have ever smoked (current and ex-smokers), total cumulative exposure was estimated with pack-years: one pack-year equals cigarettes per day multiplied by number of years smoked divided by 20 . In addition, disease variables were recorded including: classification as AS, symptom duration, duration since diagnosis, HLA-B27 status if available and extra-axial disease features (peripheral joint involvement, psoriasis, uveitis and inflammatory bowel disease). HLA-B27 was not systematically tested in this real-life cohort and therefore was missing in a significant proportion. Current use of non-steroidal anti-inflammatory drugs (NSAIDs) and tumour necrosis factor inhibition (TNFi) was also recorded. Disease activity and functional status were assessed using numeric rating scale versions of the Bath AS Disease Activity Index (BASDAI), spinal pain (spNRS) and Bath AS Functional Index (BASFI). Patient global was introduced into clinical practice after study commencement and was therefore incomplete with data missing at random. Blood samples were taken on the day of assessment, for analysis of erythrocyte sedimentation rate (ESR) and C-reactive protein (CRP). Ankylosing Spondylitis Disease Activity Score (ASDAS) was calculated using CRP for patients with complete data [14].

Statistical analyses were performed using Stata12. Comparison of patient and disease characteristics between the three smoking categories were performed using ANOVA for Gaussian, Kruskal-Wallis test (or Mann-Whitney $U$ if two categories) for non-Gaussian and Chi-squared or Fisher's exact test for categorical variables. Multivariable linear regression models were used to explore the association between each measure of disease activity (BASDAI, ASDAS, spNRS, BASFI, CRP and ESR) in turn as the dependent variable, and non-/ever-smoking as the dichotomous independent variable, adjusted for age, gender and use of TNFi. Symptom duration was not included as a covariate, given its collinearity with age. Due to their non-Gaussian distribution, ESR and CRP were log-transformed prior to regression $(\ln (\mathrm{ESR}), \ln (\mathrm{CRP}))$. ASDAS was regressed using complete case analysis and again using multiple imputation for those with missing patient global scores. Multiple imputation was performed using multivariate normal distribution with 30 imputed datasets. Variables used in the imputation model were those specified in the regression models, with BASDAI in addition as an auxiliary variable [15]. The aim of this study was to explore associations between smoking and disease activity. As patients with both AS and nr-axSpA can manifest similar levels of disease activity [3], these patients were grouped together in the analyses.

To explore the effect of pack-years on the above markers of disease severity, the same multivariable linear models were used, with pack-years as the independent variable categorised into four groups $(<10,11-20,21-40$ and $>40$ pack-years) and analysed as dummy variables with $<10$ pack-years as the reference. Categorisation was arbitrary to give similar groups sizes. Results were presented as coefficients and $95 \%$ confidence intervals (95\% CI). Residuals from each model were tested against normal distribution using Shapiro-Francia test.

This study received UK Research Ethics Committee approval (15/LO/1519).

\section{Results}

The study recruited 238 patients with established axSpA. The cohort was predominantly male $(76 \%)$ with mean age of 46.4 years $(\mathrm{SD} \pm 13.7$ ), median symptom duration of 17.1 years [inter-quartile rage (IQR) 8.4, 29.3] and median duration since diagnosis of 5.0 years [IQR $0.8,14.8$ ]. HLAB27 was measured in $61 \%$ of the cohort and of these $61 \%$ were positive. AS was present in $83 \%$. Use of NSAIDs was reported by $163(68 \%)$ patients, and $79(33 \%)$ were treated with TNFi.

At the time of assessment, a history of ever-smoking was reported by $112(47 \%)$ patients with $78(33 \%)$ reporting current smoking. Among ever-smokers, the median pack-year was 20 [IQR 10, 30]. The mean age of ex-smokers was older at 52 years, with similar ages of 
Table 1 Patient and disease characteristics of the cohort and for each smoking category

\begin{tabular}{|c|c|c|c|c|c|}
\hline & All & Non-smokers & Ex-smokers & Current smokers & $P$ value \\
\hline Number of patients & 238 & $126(52.9 \%)$ & $34(14.3 \%)$ & $78(32.8 \%)$ & \\
\hline Age & $46.4(13.8)$ & $46.1(14.2)$ & $51.7(12.1)$ & $44.6(13.2)$ & 0.046 \\
\hline Male gender & $180(75.6 \%)$ & $87(69.1 \%)$ & $31(91.2 \%)$ & $62(79.5 \%)$ & 0.018 \\
\hline $\mathrm{BMI}^{\mathrm{a}}$ & $27.6[25.1,31.0]$ & $27.4[25.2,30.5]$ & $29.0[25.8,32.6]$ & $27.6[24.7,31.0]$ & 0.443 \\
\hline AS diagnosis & $197(82.8 \%)$ & $99(78.6 \%)$ & $27(79.4 \%)$ & $71(91.0 \%)$ & 0.062 \\
\hline HLA-B27 status ${ }^{\mathrm{a}}$ & $89(61.0 \%)$ & $49(59.8 \%)$ & $14(70.0 \%)$ & $26(59.1 \%)$ & 0.670 \\
\hline Symptom duration (years) & $16.5[8.4,28.4]$ & $15.0[6.4,30.0]$ & $23.1[12.6,33.1]$ & $16.3[8.8 .26 .7]$ & 0.033 \\
\hline Diagnosis duration (years) & $5.0[0.8,14.8]$ & $4.7[0.8,15.2]$ & $4.0[1.2,14.8]$ & $0.9[5.4,14.4]$ & 0.802 \\
\hline Pack-years & $0[0,15]$ & 0 & $20[10,30]$ & $15[9,30]$ & $0.151^{b}$ \\
\hline BASDAI & $5.7[3.3,7.6]$ & $5.2[3.0,7.5]$ & $6.0[4.1,8.2]$ & $6.5[3.6,7.6]$ & 0.132 \\
\hline Spinal pain & $6.0[3.0,8.0]$ & $5.0[2.0,8.0]$ & $7.0[3.0,8.0]$ & $7.0[3.0,8.0]$ & 0.254 \\
\hline $\operatorname{ASDAS}^{\mathrm{a}}$ & $2.70(1.14)$ & $2.39(1.16)$ & $3.28(1.08)$ & $2.96(0.98)$ & 0.001 \\
\hline BASFI & $5.7[2.9,7.6]$ & $5.0[2.3,7.4]$ & $6.9[3.1,8.1]$ & $5.9[3.2,7.7]$ & 0.116 \\
\hline $\mathrm{CRP}\left(\mathrm{mg} / \mathrm{l}^{\mathrm{a}}\right.$ & $3[1,9]$ & $3[1,8]$ & $5[2,11]$ & $5[1,11]$ & 0.131 \\
\hline $\operatorname{ESR}(\mathrm{mm} / \mathrm{hr})^{\mathrm{a}}$ & $8[5,21]$ & $8[5,19]$ & $10[6,26]$ & $10[5,26]$ & 0.123 \\
\hline Peripheral joint involvement & $55(23.6 \%)$ & $35(28.0 \%)$ & $8(23.5 \%)$ & $12(16.2 \%)$ & 0.167 \\
\hline Psoriasis & $39(16.4 \%)$ & $21(16.70 \%)$ & $6(17.7 \%)$ & $12(15.4 \%)$ & 0.949 \\
\hline Uveitis & $64(26.9 \%)$ & $38(30.2 \%)$ & $12(35.3 \%)$ & $14(18.0 \%)$ & 0.079 \\
\hline IBD & $22(9.2 \%)$ & $14(11.1 \%)$ & $3(8.8 \%)$ & $5(6.4 \%)$ & 0.576 \\
\hline TNFi & $79(33.2 \%)$ & $39(31.0 \%)$ & $12(35.3 \%)$ & $28(35.9 \%)$ & 0.737 \\
\hline NSAID & $163(68.5 \%)$ & $87(69.1 \%)$ & $20(58.8 \%)$ & $56(71.8 \%)$ & 0.390 \\
\hline
\end{tabular}

Data are presented in $n(\%)$, mean (SD), median [IQR] and comparison used Chi-squared/Fisher's exact, ANOVA and Kruskal-Wallis tests, respectively

axSpA axial spondyloarthritis, ASDAS Ankylosing Spondylitis Disease Activity Score, $B M I$ body mass index, $I B D$ inflammatory bowel disease, TNFi TNF inhibition therapy, NSAID non-steroidal anti-inflammatory drugs

a BMI complete data in 190; HLAB27 status known in 146; ASDAS in 188; CRP in 231; ESR in 230

b Mann-Whitney $U$ test

current smokers at 45 years and non-smokers at 46 years. Similarly, symptom duration was longest in the ex-smoker group at 23 years. There were also more males (91\%) in the ex-smoking group than other groups. The median BASDAI was 5.7 [IQR 3.3, 7.6] and BASFI 5.7 [3.3, 7.6]. ASDAS was available for $188(79 \%)$ patients with mean of 2.7 $(\mathrm{SD} \pm 1.14)$. Patient demographics and disease characteristics compared between smoking categories are shown in Table 1.

No significant differences were seen between smoking groups for duration since diagnosis. Proportion of AS was higher in current smokers compared with rest of the cohort (91 vs. $79 \%, P=0.019$ ).

Extra-axial features were similarly prevalent between the three smoking categories. However, the prevalence of uveitis was lower in current smokers compared to rest of the cohort (18 vs. $31 \%, P=0.03$ ).

Median disease severity measures were all higher in current and ex-smokers than non-smokers, but there were no statistically significant differences between the three smoking categories. Compared to non-smokers, ever-smokers had significantly higher BASDAI, ASDAS and BASFI, but not spNRS, ESR or CRP (data not shown). The use of TNFi and NSAIDs was similar between three groups.

In all multivariable models, no significant interactions were found between independent variables. NSAID-use and HLA-B27 were not included as covariates as they did not demonstrate significant association in, or improvement to, the models. In multivariable linear regression models, ever-smoking was independently associated with higher BASDAI $(\beta=0.91,95 \%$ CI $0.26,1.55)$, spNRS $(\beta=0.85$, $95 \%$ CI $0.11,1.59)$ and BASFI $(\beta=0.82,95 \%$ CI 0.10 , 1.53). Ever-smoking was also associated with ASDAS using complete case analysis $(\beta=0.70,95 \%$ CI $0.39,1.01)$ and multiply imputed data ( $\beta=0.71,95 \%$ CI $0.40,1.01)$. However, ever-smoking was not associated with CRP $(\beta=2.68,95 \% \mathrm{CI}-1.85,7.21)$ or $\operatorname{ESR}(\beta=4.34,95 \% \mathrm{CI}$ $-0.91,9.58)$.

Multivariable linear models exploring associations with pack-year categories demonstrated significant dose-related increase for BASDAI, ASDAS and BASFI but not spNRS, CRP or ESR (Table 2). Compared to the $<10$ pack-year 
Table 2 Multivariable linear regression models of association between pack-year categories and measures of disease activity (adjusted for age, gender and use of TNFi)

\begin{tabular}{lllll}
\hline & $<10$ pack-years $n=40$ & $11-20$ pack-years $n=34$ & $21-40$ pack-years $n=32$ & $>40$ pack-years $n=6$ \\
\hline BASDAI & Reference & $0.55(-0.55,1.65)$ & $\mathbf{1 . 6 2}(\mathbf{0 . 2 8 , 2 . 9 5})$ & $\mathbf{2 . 6 1}(\mathbf{0 . 4 2}, \mathbf{4 . 8 0})$ \\
ASDAS complete case analysis & Reference & $0.31(-0.23,0.84)$ & $\mathbf{0 . 8 2}(\mathbf{0 . 1 4 , \mathbf { 1 . 5 1 } )}$ & $0.54(-0.53,1.62)$ \\
ASDAS multiply imputed & Reference & $0.33(-0.20,0.86)$ & $\mathbf{0 . 9 2}(\mathbf{0 . 2 5}, \mathbf{1 . 5 9})$ & $0.88(-17,1.93)$ \\
Spinal pain & Reference & $0.54(-0.77,1.85)$ & $1.34(-0.25,2.93)$ & $1.40(-1.21,4.01)$ \\
BASFI & Reference & $0.95(-0.29,2.20)$ & $\mathbf{2 . 0 5}(\mathbf{0 . 5 4 , 3 . 5 6 )}$ & $\mathbf{3 . 2 4}(\mathbf{0 . 7 6 , 5 . 7 1 )}$ \\
CRP & Reference & $0.08(-0.56,0.72)$ & $0.53(-0.24,1.30)$ & $1.08(-0.22,2.37)$ \\
ESR & Reference & $0.27(-0.26,0.80)$ & $0.28(-0.38,0.93)$ & $0.71(-0.33,1.76)$ \\
\hline
\end{tabular}

Statistically significant values are represented in bold

Results are presented as $\beta$ coefficient (95\% confidence interval)

BASDAI Bath Ankylosing Spondylitis Disease Activity Index, ASDAS Ankylosing Spondylitis Disease Activity Score, BASFI Bath AS Functional Index, CRP C-reactive protein, ESR erythrocyte sedimentation rate

reference group, smokers with 11-20 pack-years did not have significantly higher BASDAI or BASFI. However, heavier smokers with 21-40 pack-years had scores 1.6 higher for BASDAI and 2.1 higher for BASFI than the reference group. The group with $>40$ pack-year exposure had scores 2.6 and 3.2 higher than the reference group for BASDAI and BASFI, respectively. Pack-years were also associated with ASDAS; however, this was not significant in the highest smoking category. Smokers with 21-40 pack-years had nearly one point higher ASDAS than the reference group. Residuals from all regression models were not statistically different from the normal distribution.

\section{Discussion}

This cross-sectional study of an axSpA cohort has demonstrated that smoking is associated with increased disease activity and functional impairment. In axSpA participants reporting history of ever-smoking, increased cumulative smoking exposure was observed to have a clear independent and significant association with BASDAI and BASFI. This was irrespective of whether participants were current or ex-smokers. Compared to light smoking exposures $(<10$ pack-years), those with higher pack-year smoking histories had increased BASDAI and BASFI. Ever-smokers with 21-40 pack-year history had BASDAI and BASFI approximately two points higher than light smokers. Scores were highest in those with more than 40 pack-years of smoking.

A trend was also demonstrated for the more sensitive index ASDAS. The loss of statistical significance for the heaviest pack-year category is likely due to the small size of this group. No significant associations were demonstrated for ESR or CRP. The effect sizes increased with increasing pack-year categories; therefore, it may be that statistical power was insufficient. CRP and ESR are not specific markers of axSpA disease activity and may be influenced by other factors.

These findings are in agreement with three of the six existing studies of pack-years in AS [7, 8, 13]. Chen et al. [8] reported unadjusted correlation between pack-years and BASFI, but not BASDAI, ESR or CRP. An Iranian study found pack-years to be associated with BASDAI and of quality of life (QoL) but not BASMI in cohort of very light current smokers (median of 0.6 pack-years) [13]. The largest and most robust study found associations between categories of pack-years and all of their markers including BASDAI, BASFI, pain numerical rating score and two measures of QoL [7].

The three remaining studies reported no associations between markers of disease severity and pack-years [11, 12, 16]. There are several possible explanations for the inconsistent results. Firstly, it is important to consider confounders when studying smoking and disease activity. Some studies used simple correlation $[8,16]$ and others used variable approaches when selecting covariates $[12,13]$. Secondly, most studies recruited smaller cohorts with fewer (35-84 patients) entered into pack-year analysis $[8,11-13]$. The larger studies were also not without limitations: one was survey-based with $64 \%$ response rate to postal survey and no adjustment for TNFi [7] and the other explored association with BASFI alone with unadjusted correlation [16]. Our study is the first to quantify effect size of the association between pack-years and markers of disease activity using multi-adjusted analysis.

Our finding that only heavier smokers ( $>20$ pack-years) had significantly higher disease activity was also observed by Mattey et al. [7] In their survey study of AS patients using a test for trend, BASDAI, BASFI, pain and two QoL measures were all associated with higher smoking exposure 
(>15 pack-years), but not for those with $\leq 15$ pack-years. This is more likely as a result of statistical rather than biological effects.

In our axSpA cohort, those with a history of ever-smoking had more severe disease than non-smoking participants. This was in agreement with previous studies $[5,7,8,11]$. Ex-smokers were older and had longer symptom duration than current or non-smokers. This was also observed by Mattey et al. and may be that smoking tends to start at a younger age.

AS was more prevalent in current than non-current smokers. This could not be explained by age or gender (factors associated with radiographic progression); current smokers were younger with shorter disease and symptom duration and did not have more males than other groups. Other studies have reported greater radiographic progression in AS smokers [9, 10, 12].

It was also interesting to observe that uveitis was reported less frequently in current smokers, compared to ex-/non-smokers. Cigarette smoking is a recognised risk factor for uveitis in the general population [17]. However, development of the extra-articular disease manifestation may trigger smoking cessation behaviour. This finding was also reported in a larger Scottish cohort, where the authors hypothesised that cigarette smoke may be an irritant in those with uveitis leading to increased rates of cessation, or that the effect of uveitis on quality of life may have a particular impact on health behaviour [18]. This may also be one of many risk factor paradoxes due to collider-stratification bias described in detail in 19.

The disparity between symptom and disease duration in this cohort suggests a prolonged delay to referral to the SpA service for diagnosis. This reflects the ongoing need to raise awareness of axSpA in primary care. Continuous NSAIDs was not promoted at this spondyloarthritis service given side effects and uncertain benefit [20]. Patients in this cohort generally took NSAIDs on-demand, and NSAID index was therefore not used.

There were several limitations to this study. The crosssectional design limits ability to infer causality between smoking and disease severity. However, the clear doseresponse relationship provides some support for smoking causing more severe disease phenotype in axSpA. Studies of smoking status are always subject to social desirability bias. It is possible that patients are misclassified by self-reported smoking status. This is especially true for ex- and current-smoking groups and is one reason that they were analysed together under ever-smokers. Future studies should set more stringent definitions of ex-smoking. This will allow separately analysis of dose-response relationship in ex- and current smokers. The categorisation of pack-years left only a small subgroup with heaviest (>40) pack-year exposure. Importantly, due to limited data collection, no adjustment was made for socioeconomic class in this study, although pack-years have been associated with disease activity independent of socioeconomic class [7, 18].

In conclusion, this study found that cigarette smoking was associated with increased axSpA severity in a clear dose-response manner. This dose-response relationship persists even in those who have quit smoking. Therefore, smoking cessation may be more beneficial if implemented before accruing a large number of pack-years. It is important for clinicians to help patients with smoking cessation and smoking reduction as early as possible.

Acknowledgements We thank research nurses Helen Frankland and Ayren Mediana for their help in recruiting patients and collecting data. We also thank Stephen Duffield for proofreading and helpful comments.

Funding No funding was received for this study.

\section{Compliance with ethical standards}

Conflict of interest The authors declare no conflicts of interest.

Ethical approval All procedures performed in studies involving human participants were in accordance with the ethical standards of the institutional and/or national research committee and with the 1964 Helsinki Declaration and its later amendments or comparable ethical standards.

Informed consent Informed consent was obtained from all individual participants included in the study.

Open Access This article is distributed under the terms of the Creative Commons Attribution 4.0 International License (http://creativecommons.org/licenses/by/4.0/), which permits unrestricted use, distribution, and reproduction in any medium, provided you give appropriate credit to the original author(s) and the source, provide a link to the Creative Commons license, and indicate if changes were made.

\section{References}

1. van der Linden S, Valkenburg HA, Cats A (1984) Evaluation of diagnostic criteria for ankylosing spondylitis. A proposal for modification of the New York criteria. Arthritis Rheum 27(4):361-368

2. Rudwaleit M, Jurik AG, Hermann KG, Landewe R, van der Heijde D, Baraliakos X, Marzo-Ortega H, Ostergaard M, Braun J, Sieper J (2009) Defining active sacroiliitis on magnetic resonance imaging (MRI) for classification of axial spondyloarthritis: a consensual approach by the ASAS/OMERACT MRI group. Ann Rheum Dis 68(10):1520-1527. doi:10.1136/ $\operatorname{ard} .2009 .110767$

3. Baraliakos X, Braun J (2015) Non-radiographic axial spondyloarthritis and ankylosing spondylitis: what are the similarities and differences? RMD Open 1(Suppl 1):e000053. doi:10.1136/ rmdopen-2015-000053 
4. Videm V, Cortes A, Thomas R, Brown MA (2014) Current smoking is associated with incident ankylosing spondylitis-the HUNT population-based Norwegian health study. J Rheumatol 41(10):2041-2048. doi:10.3899/jrheum.140353

5. Chung HY, Machado P, van der Heijde D, D'Agostino MA, Dougados M (2012) Smokers in early axial spondyloarthritis have earlier disease onset, more disease activity, inflammation and damage, and poorer function and health-related quality of life: results from the DESIR cohort. Ann Rheum Dis 71(6):809-816. doi:10.1136/annrheumdis-2011-200180

6. Wendling D, Prati C (2013) Spondyloarthritis and smoking: towards a new insight into the disease. Expert Rev Clin Immunol 9(6):511-516. doi:10.1586/eci.13.35

7. Mattey DL, Dawson SR, Healey EL, Packham JC (2011) Relationship between smoking and patient-reported measures of disease outcome in ankylosing spondylitis. J Rheumatol 38(12):2608-2615. doi:10.3899/jrheum.110641

8. Chen $\mathrm{CH}$, Chen HA, Lu CL, Liao HT, Liu CH, Tsai CY, Chou CT (2013) Association of cigarette smoking with Chinese ankylosing spondylitis patients in Taiwan: a poor disease outcome in systemic inflammation, functional ability, and physical mobility. Clin Rheumatol 32(5):659-663. doi:10.1007/s10067-013-2165-y

9. Haroon N, Inman RD, Learch TJ, Weisman MH, Lee M, Rahbar MH, Ward MM, Reveille JD, Gensler LS (2013) The impact of tumor necrosis factor alpha inhibitors on radiographic progression in ankylosing spondylitis. Arthritis Rheum 65(10):26452654. doi: $10.1002 /$ art. 38070

10. Klingberg E, Nurkkala M, Carlsten H, Forsblad-d'Elia H (2014) Biomarkers of bone metabolism in ankylosing spondylitis in relation to osteoproliferation and osteoporosis. J Rheumatol 41(7):1349-1356. doi:10.3899/jrheum.131199

11. Reed MD, Dharmage S, Boers A, Martin BJ, Buchanan RR, Schachna L (2008) Ankylosing spondylitis: an Australian experience. Intern Med J 38(5):321-327. doi:10.1111/j.1445-5994.2007.01471.x

12. Sakellariou GT, Anastasilakis AD, Kenanidis E, Potoupnis M, Tsiridis E, Savvidis M, Kartalis N, Sayegh FE (2015) The effect of smoking on clinical and radiographic variables, and acute phase reactants in patients with ankylosing spondylitis. Rheumatol Int 35(12):2109-2114. doi:10.1007/s00296-015-3381-3

13. Fallahi S, Jamshidi AR, Gharibdoost F, Mahmoudi M, Ahmadzadeh N, Nicknam MH (2013) The correlation between pack-years of smoking and disease activity, quality of life, spinal mobility, and sacroiliitis grading in patients with ankylosing spondylitis. Turk J Rheumatol 28(3):181-188. doi:10.5606/tjr.2013.3269

14. Lukas C, Landewe R, Sieper J, Dougados M, Davis J, Braun J, van der Linden S, van der Heijde D, Assessment of SpondyloArthritis international S (2009) Development of an ASASendorsed disease activity score (ASDAS) in patients with ankylosing spondylitis. Ann Rheum Dis 68(1):18-24. doi:10.1136/ ard.2008.094870

15. Young R, Johnson D (2011) Imputing the missing Y's: implications for survey producers and survey users. Proceedings of the AAPOR conference abstracts, pp 6242-6248

16. Ward MM, Weisman MH, Davis JC Jr, Reveille JD (2005) Risk factors for functional limitations in patients with long-standing ankylosing spondylitis. Arthritis Rheum 53(5):710-717. doi:10.1002/art.21444

17. Roesel M, Ruttig A, Schumacher C, Heinz C, Heiligenhaus A (2011) Smoking complicates the course of non-infectious uveitis. Graefes Arch Clin Exp Ophthalmol 249(6):903-907. doi:10.1007/s00417-010-1597-1

18. Jones GT, Ratz T, Dean LE, Macfarlane GJ, Atzeni F (2014) The effect of smoking cessation in ankylosing spondylitis-results from the Scotland Registry for Ankylosing Spondylitis (SIRAS). Clin Exp Rheumatol 32:773

19. Choi HK, Nguyen US, Niu J, Danaei G, Zhang Y (2014) Selection bias in rheumatic disease research. Nat Rev Rheumatol 10(7):403-412. doi:10.1038/nrrheum.2014.36

20. Kroon FP, van der Burg LR, Ramiro S, Landewe RB, Buchbinder R, Falzon L, van der Heijde D (2015) Non-steroidal anti-inflammatory drugs (NSAIDs) for axial spondyloarthritis (ankylosing spondylitis and non-radiographic axial spondyloarthritis). Cochrane Database Syst Rev 7:CD010952. doi:10.1002/14651858.CD010952.pub2 